\title{
Driver eXperience (DX): Una aproximación a la interacción en el vehículo
}

\author{
Driver eXperience (DX): An approach to the in-vehicle interaction
}

\author{
Juan S. Guzmán ${ }^{1}$ (D), Isabela Toledo ${ }^{1}$ (D) , Huizilopoztli Luna-García ${ }^{(D)}$, César Alberto Collazos ${ }^{1}$ \\ ${ }^{1}$ Universidad del Cauca, Popayán, Colombia \\ ${ }^{2}$ Universidad Autónoma de Zacatecas, Zacatecas, México \\ juanseguzman@unicauca.edu.co, tbisabela@unicauca.edu.co,hlugar@uaz.edu.mx,ccollazo@unicauca.edu.co
}

(Recibido: 21 Septiembre 2020; aceptado: 25 Octubre 2020; Publicado en Internet: 1 Diciembre 2020)

\begin{abstract}
Resumen. La Experiencia de Usuario (UX) ha tenido un crecimiento en diferentes campos de la industria automotriz. Los vehículos se han convertido en un mundo lleno de oportunidades que le permiten al conductor sumergirse en un entorno de conectividad, comunicación, información y entretenimiento. Por lo tanto, es importante comenzar a darle al usuario un estudio más contextualizado, en donde no sea tratado como un individuo en general, sino más bien, por lo que realmente es, un conductor, con atributos y acciones propias de su rol en el vehículo. Este artículo presenta una definición subjetiva del término Driver eXperience (DX), y un conjunto de factores para tener en cuenta a fin de mejorar la relación vehículo-conductor, y así, brindarle al usuario una aproximación más comprensiva. Adicionalmente, se presenta un mapeo de la literatura de UX en la industria automotriz para entender la relevancia que puede tener introducir el término de DX.
\end{abstract}

Palabras clave: Experiencia de Usuario, Experiencia del Conductor, Interacción en el vehículo, Industria automotriz.

Abstract. User eXperience (UX) has been a growing area in different fields in the automotive industry. Vehicles have become a world full of opportunities that allow drivers to immerse themselves in an environment of connectivity, communication, information, and entertainment. Thus, it is important to start giving the user a more contextualized study, where he/she is not treated as a general individual, but instead for what he/she is, a driver, with specific features and actions. This paper presents a subjective definition of Driver eXperience (DX) and the factors to take into account to enhance the vehicle-driver relationship and therefore bring the driver a more comprehensive approach when performing in-vehicle tasks. Additionally, this paper presents a literature mapping of UX in the automotive field, intending to understand the relevance that could have the introduction of the DX term.

Keywords: User eXperience, Driver eXperience, In-vehicle interaction, Automotive industry.

Tipo de artículo: Artículo de revisión.

\section{Introducción}

A lo largo de los años, el término Experiencia de Usuario o UX (acrónimo de User eXperience en inglés) ha sido muy debatido y definido de múltiples formas (Hellweger \& Wang, 2015). Al igual que otros conceptos en la línea de investigación de Interacción Humano-Computador, UX se ha especificado desde diversas perspectivas y ha sido utilizado para enfatizar en distintos aspectos según el contexto. Podemos definir este término en un nivel muy alto como un concepto que incluye todos los aspectos relacionados con el cómo los individuos interactúan con un producto (Borchers \& Thomas, 2001; Hassenzahl \& Tractinsky, 2006). La investigación en UX ha incrementado en diferentes campos, contribuyendo a la mejora de productos software, generación de valor a los clientes de una empresa, interacción con una aplicación, entre otros (Blackwell \& Fincher, 2010; van Schaik \& Ling, 2008). Una de las industrias que ha estado implementado técnicas de diseño de UX para mejorar la interacción del usuario, es el campo automotriz (Kaiser et al., 2016).

Hoy en día, los vehículos se han convertido en un mundo lleno de opciones que permiten a los conductores sumergirse en un entorno de conectividad, comunicación, información y entretenimiento (Boehm-Davis et al., 2003). La industria automotriz ha tenido un crecimiento en aspectos como vehículos autónomos, sistemas infotainment, sistemas de reconocimiento de gestos, entre muchas otras tecnologías 
(Shimizu, 2017), que cada día brindan al usuario un mayor número de oportunidades al conducir, y al mismo tiempo, un mayor número de elementos que deben analizarse para brindar una mejor experiencia en el vehículo. Por tanto, puede resultar útil empezar a introducir el término Experiencia del Conductor o DX (acrónimo de Driver eXperience en inglés) para establecer un conjunto de factores contextualizados que permitan a los investigadores de la industria evaluar la interacción hombre-vehículo de una forma más exhaustiva y específica, teniendo en cuenta como principal objeto de estudio el conductor y los atributos y acciones que definen su rol en el vehículo.

Este artículo tiene como objetivo presentar un mapeo científico de la evolución de la literatura relacionada con la experiencia del usuario en la industria automotriz para comprender la relevancia de introducir el término DX, así como su definición subjetiva y factores importantes a tener en cuenta para evaluar dicha relación vehículo-conductor.

\section{Antecedentes}

Como se menciona, el término de UX ha sido definido desde distintas perspectivas por múltiples autores a lo largo del tiempo, en un esfuerzo por identificar los factores y dimensiones a considerar para evaluar la experiencia del usuario, lo que nos permite inferir que se trata de un concepto dinámico y dependiente del contexto (Bosley, 2013; Law et al., 2014). Alben (1996) afirma que UX comprende todos los aspectos de cómo las personas usan un producto interactivo, cómo se siente en sus manos, qué tan bien entienden cómo funciona, cómo se sienten al respecto mientras lo usan, qué tan bien sirve sus propósitos, y qué tan bien encaja en todo el contexto en el que lo están usando. Del mismo modo, Hassenzahl \& Tractinsky (2006) argumentan que UX es una consecuencia del estado interno del usuario, las características del sistema diseñado y el contexto (o el entorno) dentro del cual ocurre la interacción. Autores destacados como Norman \& Nielsen (2014) sostienen que el término de UX abarca todos los aspectos de la interacción del usuario final con la empresa, sus servicios y productos, y dan a entender que el principal requisito para brindar una experiencia ejemplar es satisfacer las necesidades exactas del cliente. Otros factores que toman en cuenta estos autores son la simplicidad y la elegancia que producen los productos. En otras definiciones recientes como la de Ross (2014) se establece que UX hace referencia al "sentimiento o experiencia general que una persona tiene cuando usa un producto, como un sitio web, un dispositivo móvil o una aplicación de software. Incluye lo fácil que es de usar, lo agradable y satisfactoria que es la experiencia, si es útil o no y cuán bien satisface las necesidades de la persona”. UX también se puede definir como la respuesta sensorial, emocional y reflexiva del usuario a la interacción con un sistema en un contexto, donde este usuario se refiere a la persona que usa o emplea algo, el sistema indica el dispositivo u objeto artificial que está organizado para un propósito, el contexto introduce las condiciones interrelacionadas en las que algo existe u ocurre, y la interacción señala la cadena de acciones y reacciones interdependientes entre un usuario y un sistema. Además, algunos enfoques destacan la importancia de tener en cuenta elementos como la temporalidad para fortalecer la relación a largo plazo entre el usuario y el sistema (Karapanos et al., 2009; Tscheligi, 2012). Todos estos componentes de UX se pueden especificar y contextualizar, llevando su definición a la relación conductor-vehículo en un esfuerzo por comenzar a introducir el término DX. La introducción de este concepto no busca separarlo de su raíz central, es decir UX, sino más bien, enfocarlo e instanciar sus componentes, en búsqueda de obtener un diseño de la experiencia más centrado en el usuario por sus atributos y acciones propias que lo definen como conductor y lo diferencian de otro tipo de individuos.

\section{Mapeo de la revisión de literatura}

Con el objetivo de identificar la relevancia de una introducción del término DX, se procede a realizar una revisión de literatura que permita conocer la evolución que ha tenido la experiencia de usuario en diversos campos de la industria automotriz. Para realizar el análisis sistemático de la literatura, se hace uso de la propuesta metodológica de Medina-Lopez et al. (2010) en donde se especifican las fases a realizar para cumplir con la revisión. Las fases mencionadas corresponden a las siguientes: (I) Identificación del campo de estudio y periodo a analizar, (II) Selección de las fuentes de información, (III) Realización de la búsqueda, (IV) Gestión y depuración de los resultados y (V) Análisis de los resultados. Cada una de las 
actividades fue realizada de manera ordenada y cronológica, obteniendo los resultados necesarios para los objetivos de este trabajo. Para la primera fase, se decidió delimitar la base conceptual a estudiar, tomando como objeto de investigación los trabajos relacionados con el término UX aplicado en algún sector de la industria automotriz. De igual manera, se acotó el periodo de estudio a 2012 como año mínimo de publicación de los trabajos, para lograr visualizar la evolución del estudio de estos factores de interacción en el vehículo en un contexto más cercano temporalmente. Para la segunda fase, se hizo selección de Scopus como la base de datos bibliográfica a explorar. En la realización de la búsqueda, es decir la tercera fase, se obtuvieron 2362 documentos que contaban con la intersección de "UX" con términos relacionados a la industria automotriz como "driver", "vehicle", "automobile" o "driving" en su título, palabras claves y/o resumen, con el objetivo de encontrar los trabajos que se enfocaran en una investigación relacionada con la experiencia de usuario aplicada en los vehículos. Luego de ello, se procedió a descartar un número de artículos cuya cantidad de citaciones fuera cero y su año de publicación fuera menor al año mínimo establecido en la fase II, resultando en un total de 1725 documentos. Para la cuarta fase, en donde se pretende realizar una gestión de los artículos seleccionados y obtener resultados a partir de ello, se hace uso de SciMAT. SciMAT es una herramienta de código libre que permite realizar análisis de mapas científicos en un marco longitudinal, y cuantificar los resultados mediante indicadores bibliométricos (Cobo et al., 2012). Esta herramienta incorpora métodos, algoritmos y métricas para todos los pasos del flujo de trabajo del mapeo científico, desde el preprocesamiento hasta la visualización de los resultados.

Considerando el objetivo de este documento, se procede a generar un conjunto de diagramas y redes temáticas con base en los artículos seleccionados, para comprender la evolución que han tenido las técnicas de UX en los vehículos y dar a conocer la relevancia de introducir un término que encamine la definición de sus factores de estudio en torno a la experiencia del conductor. Para la generación de estos componentes de visualización, SciMAT permite efectuar un análisis estratégico, el cuál sitúa cada red temática en una posición relativa dentro de la red global de acuerdo con sus valores de cohesión externa o centralidad, y la cohesión interna o densidad. En primer lugar, para efectuar dicho procedimiento, se identificaron y clasificaron las palabras claves más utilizadas y relevantes en los artículos, lo cuál iba a permitir generar esos núcleos temáticos encaminados a darle sentido al análisis. Posteriormente, este conjunto de palabras claves seleccionadas o temas, fueron caracterizados de acuerdo con las métricas mencionadas: centralidad y densidad. La centralidad mide el grado de fuerza de los enlaces externos del tema con otros temas. Esta medida se puede interpretar como la importancia de un tema en el desarrollo global de campo científico analizado, o como el grado de cohesión externa del tema. Por otro lado, la densidad mide la fuerza interna de todos los enlaces entre las palabras clave que describen al tema, o, dicho de otro modo, el grado de cohesión interna. SciMAT, hace uso de las medidas de tendencia central de estas dos métricas en cada uno de los temas para clasificarlos en cuatro categorías y posicionarlos en un espacio bidimensional, el cuál se denomina diagrama estratégico. Estas cuatro categorías corresponden a (1) temas motores, (2) temas muy desarrollados y aislados, (3) temas emergentes o en desaparición, y (4) temas básicos y transversales.

Para este caso, se decide segmentar los artículos en tres grupos dependiendo de su fecha de publicación, con el objetivo de visualizar la evolución literaria esperada y así reconocer el comportamiento de los núcleos temáticos en el tiempo. Esta segmentación temporal se realiza de la siguiente forma: 2012-2014, 2015-2017 y 2018-2020. Luego de realizar las actividades mencionadas, se procede a desplegar finalmente el diagrama estratégico que se logra observar en la Figura 1.

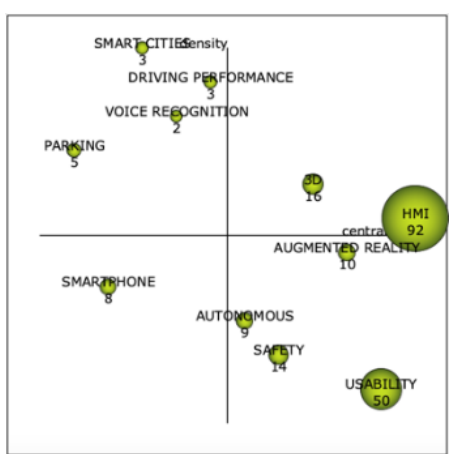

a) 2012-2014

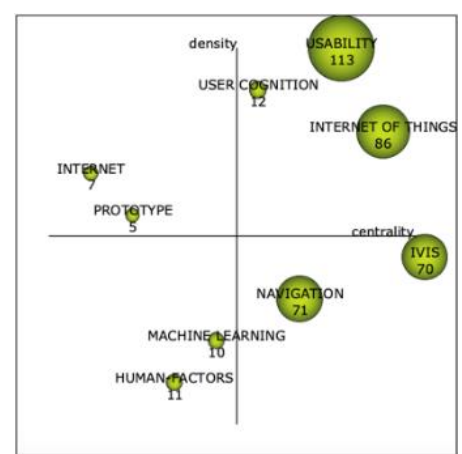

b) $2015-2017$

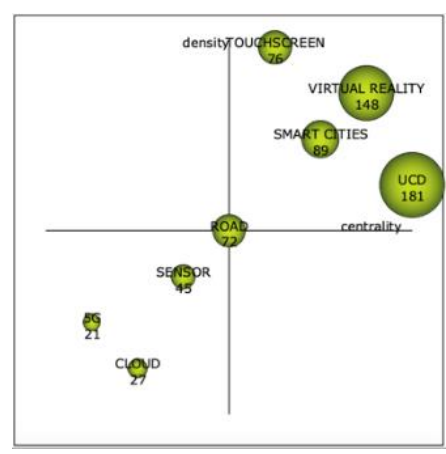

c) $2018-2020$

Figura 1. Diagrama estratégico 
Los temas motores se ubican en el cuadrante superior derecho y corresponden a los núcleos temáticos bien desarrollados e importantes en la construcción del campo científico del periodo estudiado, debido a que presentan una fuerte centralidad y una alta densidad. Cabe mencionar que estos temas se encuentran muy relacionados externamente con conceptos aplicables a otros núcleos temáticos. Los temas que se ubican en el cuadrante superior izquierdo, es decir, los temas muy desarrollados y aislados, tienen una importancia marginal en el campo científico, lo que los caracteriza por estar muy especializados y ser muy específicos. Los temas emergentes o en desaparición se ubican en el cuadrante inferior izquierdo, representando núcleos temáticos poco desarrollados y marginales, debido a que presentan una densidad y centralidad débil. Los temas que se ubican en el cuadrante inferior derecho, es decir, los temas básicos o transversales, son de gran importancia para el campo científico, sin embargo, su densidad nos da a conocer que no están bien desarrollados a comparación de los demás objetos de estudio en el periodo especificado.

Los núcleos temáticos que aparecen en la Figura 1, no representan todos los temas tratados en esos periodos, si no que tienen la intención de agrupar los conceptos y de esta forma mostrar aquellos temas que fueron más relevantes e integraban las demás palabras clave en dichos periodos con base en los artículos estudiados. También cabe mencionar que el número que aparece debajo del nombre del núcleo temático indica la cantidad de documentos que tratan dicho tema y el tamaño de la esfera es proporcional a este valor numérico. Conociendo esto, se logra observar en la Figura 1, que las Interfaces Humano-Máquina (HMI) corresponden al núcleo temático más tratado entre los años 2012 a 2014, así mismo como que la usabilidad y el Internet de las Cosas (IoT) fueron sobresalientes en el periodo de estudio siguiente. Durante los años más recientes, es decir de 2018 a 2020, se percibe que temas como las pantallas táctiles, la Realidad Virtual, el estudio de los vehículos en ciudades inteligentes y el Diseño Centrado en el Usuario (UCD), son los núcleos más relevantes en los artículos estudiados para dicho periodo.

Como fue mencionado, estos núcleos no resaltan todos los temas tratados en los años de estudio, si no que permiten centralizar los conceptos. Cada una de estas esferas de los diagramas estratégicos despliega una red que se complementa a partir de los otros temas existentes en los artículos y la relación que tienen con el tema central. Estos grafos adicionales que se despliegan se conocen como redes temáticas. Estas redes permiten conocer las relaciones de los temas dentro de los núcleos temáticos que se despliegan en el diagrama estratégico, para tener una visión más detallada de la evolución literaria y de los conceptos utilizados en los trabajos estudiados. Para este caso, y teniendo en cuenta los resultados de la Figura 1 c), podemos analizar a detalle la red temática que corresponde al Diseño Centrado en el Usuario. Este grafo se observa en la Figura 2.

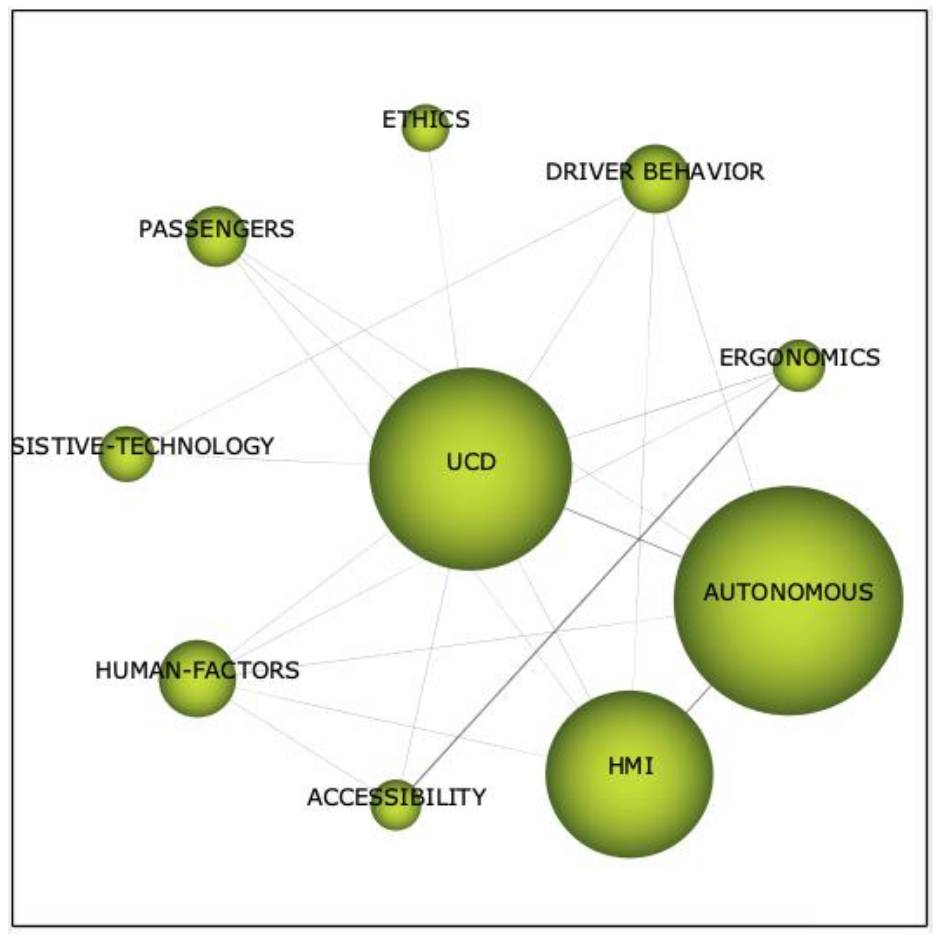

Figura 2. Red temática con UCD como núcleo 
El volumen de las esferas, al igual que en el diagrama estratégico, es proporcional al número de documentos asociados a las palabras clave del tema, y el grosor de las líneas, que representa la interrelación de los conceptos, es proporcional al índice de equivalencia, también denominado índice de asociación, que determina la probabilidad de que se mencione un tema $i$ cuando se menciona otro tema $j$ en un documento.

De la Figura 2, logramos observar que temas como vehículos autónomos, comportamiento del conductor, factores humanos, ética, entre otros, se relacionan directamente con el Diseño Centrado en el Usuario, debido a su aparición en conjunto con este último núcleo temático en diversos artículos. Se puede inferir también que temas como el diseño ergonómico y la accesibilidad presentan una relación bastante fuerte en comparación a otros, debido al grosor del vínculo que los une.

En las Figuras 1 y 2, todos los componentes visuales obtenidos en SciMAT, permiten dar a conocer esa evolución que UX ha tenido en el sector automotriz y la importancia que han tomado distintos temas en torno a mejorar la experiencia que tiene el conductor con los componentes del vehículo. Esto conlleva a demostrar la relevancia que podría tener el contextualizar los factores de UX con respecto a la relación conductor-vehículo al comenzar a mencionar el término DX y así poder contribuir en un diseño más centrado en el usuario.

\section{Driver eXperience}

Luego de conocer la relevancia de UX en la industria automotriz, procedemos a dar nuestra definición subjetiva de DX y los factores a tener en cuenta para su evaluación, con base en los enfoques de definición de UX.

DX se puede describir como la experiencia que tiene un conductor al interactuar con un vehículo y ejecutar diferentes tareas, haciendo uso de los diversos componentes y funcionalidades que este ofrece. Comprende todos los aspectos que afectan la relación conductor-vehículo y abarca lo relacionado a qué tan seguro se siente el conductor al utilizar el vehículo y sus elementos, qué tan agradable y satisfactoria es la experiencia de conducción, cómo el conductor puede realizar tareas secundarias del vehículo para mejorar su experiencia y sin ponerlo en riesgo, cuánta confianza tiene el conductor sobre el vehículo y las tecnologías integradas en este, cómo afecta al conductor el diseño del vehículo y sus componentes, cómo influye la presencia de más pasajeros sobre la experiencia del conductor y qué tan eficiente es el vehículo para satisfacer las necesidades del conductor.

A pesar de que la Experiencia de Usuario es altamente subjetiva, dinámica y dependiente del contexto, existen ciertos aspectos base subyacentes en las diversas disciplinas, que se deben tener en cuenta para describir UX como lo son: el usuario, el sistema, el contexto y la interacción (Tscheligi, 2012). Para efectos del presente trabajo, se definen dichos aspectos de forma más específica para DX, donde el usuario es lo que se conoce como el conductor, el sistema sería el vehículo, el contexto es el contexto del conductor, y la interacción son las acciones recíprocas entre conductor y vehículo.

En DX, el conductor se puede describir como la persona que realiza las tareas de conducción e interactúa con los componentes del vehículo, como el volante, los pedales, el sistema de información y entretenimiento, el tablero central, entre otros. El vehículo, es el sistema sobre el cuál el conductor interactúa y cuya función principal es cumplir satisfactoriamente las tareas que el conductor ejecuta. Por otro lado, el contexto puede referirse tanto al entorno físico en el cual el conductor interactúa con el vehículo o también a las condiciones en las que se produce la interacción. Finalmente, la interacción se puede entender como la cadena de acciones y reacciones interdependientes entre el conductor y el vehículo, como lo son la acción de conducir, tunear una estación de radio, navegar el GPS, estacionarse, entre otros.

Como se mencionó, la Experiencia de Usuario se puede contextualizar, teniendo en cuenta un conjunto de factores que podrían afectar la interacción. En este caso de estudio, se tomaron como referencia los factores de UX precisados por Tscheligi (2012) en las procedencias de la 4a Conferencia Internacional sobre Interfaces de Usuario Automotriz y Aplicaciones Vehiculares Interactivas y se adaptaron para el contexto de DX. El conjunto de factores considerados para la definición de DX son: Carga de Trabajo Mental del Conductor, Confianza del Conductor, Estética del Vehículo, Usabilidad del Vehículo, Coexperiencia del Conductor, y Seguridad Percibida por el Conductor. 


\subsection{Carga de trabajo mental del conductor}

La conducción es una actividad dinámica y compleja que implica tareas visuales, cognitivas y manuales. El conductor debe establecer objetivos estratégicos, supervisar el entorno de la carretera y los sistemas del vehículo, procesar información y elaborar planes de acción tácticos, así como ejecutar actividades de nivel de control (Michon, 1985). Conducir es claramente una tarea exigente en la que se espera que los conductores tengan altos niveles de atención en la vía y la capacidad de poder cambiar continuamente entre múltiples tareas, mientras procesa grandes cantidades de información en un entorno de carretera dinámico (Habib et al., 2019). La carga de trabajo mental se considera típicamente como la cantidad de recursos de un operador que se requieren para cumplir con las demandas de las tareas (Eggermeier et al., 1991). Así, la Carga de Trabajo Mental del Conductor es una consecuencia de la demanda de las tareas de conducción, así como de las demandas de las tareas secundarias que el conductor realice en el vehículo. La conducción implica fluctuaciones extremas en la carga de trabajo mental del conductor, donde un gran número de factores tales como la interacción con dispositivos en el vehículo, distracciones de los pasajeros y cambios en las demandas de conducción, por ejemplo, por variaciones en el tráfico y la densidad de peatones, puede afectar los niveles de atención del conductor y resultar en distracciones (Brookhuis \& de Waard, 2010). Este es un factor muy importante sobre la experiencia del conductor debido a que se ha demostrado que tanto los niveles muy altos como los muy bajos de carga de trabajo mental conducen a percepciones inexactas del entorno, bajos niveles de atención, y tiempo insuficiente para procesar la información con precisión, lo cual generalmente resulta en accidentes (da Silva, 2014).

\subsection{Confianza del conductor}

El concepto de confianza ha sido estudiado en gran variedad de disciplinas (incluyendo la psicología social, los factores humanos, y la organización industrial) para comprender las relaciones entre humanos o entre humanos y máquinas. La amplia variedad de contextos en los que se ha estudiado, conduce a diversas definiciones y teorías sobre la confianza (Lewis et al., 2018). Entre las definiciones más comúnmente aceptadas, se tiene que la confianza puede ser entendida como "La disposición de una persona para colocarse en una posición vulnerable, con respecto a una tecnología, con una expectativa positiva sobre el resultado o una naturaleza positiva del comportamiento futuro" (Mayer et al., 1995) o como "La actitud de que un agente ayudará a lograr los objetivos de un individuo en una situación caracterizada por la incertidumbre y la vulnerabilidad" (Lee \& See, 2004). Basándonos en estas ideas, la Confianza del Conductor podría describirse como la voluntad del conductor de ponerse en una situación de vulnerabilidad, con respecto al vehículo, con la expectativa de un resultado positivo. La confianza es una parte fundamental de la experiencia de conducción debido a que, el conductor espera que el automóvil responda de manera adecuada ante la ejecución de comandos y maniobras que le permitan llegar a su destino de manera segura. Asimismo, la confianza juega un papel importante para ayudar a los usuarios a superar las percepciones de riesgo e incertidumbre en el uso y aceptación de nuevas tecnologías (Gefen et al., 2003; Pavlou \& Gefen, 2004). Por esta razón, la confianza se ha vuelto un foco en el área de investigación del transporte centrado en el humano, especialmente en el diseño técnico de los futuros vehículos autónomos y ha planteado la cuestión de cómo cambian las condiciones que forman la confianza a medida que las tecnologías se vuelven más inteligentes (Raats et al., 2020).

\subsection{Estética del vehículo}

La Estética del Vehículo se refiere a la apariencia exterior y atributos del vehículo que provocan una percepción de belleza o atractivo ante los ojos del conductor. Bloch (1995) reconoce la influencia de la apariencia de un producto sobre las decisiones de compra de los consumidores, afirmando que, dada la posibilidad de elegir entre dos productos, iguales en precio y función, los consumidores compran el que consideran más atractivo. En el mercado automotriz actual, la funcionalidad y el rendimiento a menudo se dan por sentado, lo que hace que la atención de los consumidores se centre en la estética (Brown et al., 2020). Las diferentes partes y elementos del vehículo caracterizados por una combinación de elementos de diseño (como color, forma y material), impactan la experiencia del conductor, ya sea consciente o inconscientemente, desde el momento de compra y, posteriormente, mientras conduce. Para enriquecer la 
percepción que tiene el conductor sobre el vehículo, es importante diseñar para todos los sentidos, ya que las personas pueden percibir objetos y espacios con el sonido, el olfato y el tacto, así como con la vista.

\subsection{Usabilidad del vehículo}

La norma ISO 9241-11:2018 define Usabilidad como el grado en el que un sistema, producto o servicio puede ser utilizado por usuarios específicos para lograr objetivos específicos con eficacia, eficiencia y satisfacción en un contexto de uso específico (International Organization for Standardization, 2018). En general, no se puede especificar la usabilidad de un sistema (es decir, su idoneidad para un propósito) sin definir primero quiénes son los usuarios previstos del sistema, las tareas que esos usuarios realizarán con él y las características del entorno físico, organizacional y social en el que se utilizará (Brooke, 1996). Para el contexto automotriz, la Usabilidad del Vehículo se puede describir como la medida en que el conductor y otros pasajeros pueden utilizar el vehículo para realizar tareas de conducción y secundarias, logrando hacerlas con eficacia, eficiencia y satisfacción. La interacción con un sistema que es fácil de usar y comprender aumenta la productividad de los usuarios, el ritmo de aprendizaje y la satisfacción con el sistema en sí (Orlovska et al., 2018). Adicionalmente, el conductor realiza tareas secundarias dentro del vehículo, lo que muchas veces implica interactuar con un sistema de información y entretenimiento que ofrece diversas funcionalidades. Dichas funciones agregadas se diseñan con el fin de mejorar aún más la experiencia del conductor. Por lo tanto, se debe tener en cuenta que la usabilidad de las interfaces le permitan al conductor operar correctamente el sistema con una mínima distracción, facilitando su uso y manteniendo la seguridad en la vía (Li et al., 2017).

\subsection{Co-experiencia del conductor}

La co-experiencia tiene lugar cuando las experiencias se crean juntas o se comparten con otros (Forlizzi \& Battarbee, 2004). Battarbee (2004) introdujo la "co-experiencia" o "experiencia conjunta" para abordar la limitación en la literatura acerca de la carencia de aspectos sociales en la organización de la experiencia del usuario. Battarbee (2004) sostiene que es limitante ver al usuario en el contexto de los productos interactivos como un ser independiente y como consumidor pasivo de lo que el diseñador haya diseñado para ellos. Los usuarios manipulan y reorganizan sus tecnologías para que encajen con las actividades en cuestión y especialmente para apoyar la interacción y la construcción de espacios sociales. En este sentido, la Coexperiencia del Conductor se refiere a la influencia que tiene la presencia física o virtual de otros pasajeros sobre la experiencia del conductor, es decir, estudia el comportamiento del conductor en un contexto social. El diseño de los vehículos permite al conductor compartir un espacio de interacción presencial cuando invita a más pasajeros a su automóvil o también le permite hacer uso de dispositivos de información y comunicación para entablar conversaciones con otras personas a distancia que, como resultado, de una $u$ otra manera afectan la experiencia del conductor mientras maneja, ya sea positiva o negativamente.

\subsection{Seguridad percibida por el conductor}

La Seguridad Percibida por el Conductor se puede especificar como la percepción que tiene el conductor de hasta qué punto se siente seguro al interactuar con el vehículo. El diseño y la respuesta del vehículo ante diversas situaciones pueden influir en el nivel de peligro que siente el conductor al realizar tareas. La percepción que tiene el conductor de la seguridad en el entorno de conducción tiene una influencia importante sobre su comportamiento mientras conduce y en el desempeño de las tareas. La percepción de seguridad depende, en primer lugar, de la naturaleza de la información que ingresa, pero en segundo lugar y de manera muy importante, del estado emocional y las características personales del individuo (Wang et al., 2002). Un alto nivel de seguridad percibida puede ser deseable en algunas áreas, pero también puede tener efectos negativos, como que los usuarios dejen de prestar suficiente atención al realizar tareas peligrosas (Tscheligi, 2012).

Estos son los conceptos que subjetivamente consideramos los principales factores que podrían afectar el comportamiento del conductor y por lo tanto su DX en general. Contextualizar los términos de UX para el entorno automotriz puede contribuir a desarrollar estudios más centrados en el conductor, según las necesidades y las características que lo diferencian de un usuario general. Asimismo, para que el diseño y 
la evaluación de la experiencia sean más específicos para su actividad principal y su contexto, con el fin de ofrecer la más óptima experiencia para la interacción conductor-vehículo.

\section{Conclusión}

Luego de realizar un mapeo de la literatura para conocer la relevancia de la evaluación de la experiencia del usuario en la industria automotriz y la importancia que ha tomado la relación vehículo-conductor, se propuso una definición subjetiva del término DX, junto con los factores que se podrían considerar para su estudio, con el objetivo de establecer un primer acercamiento a esta contextualización del concepto UX, que podría resultar en una investigación con mayor profundidad en un futuro. La introducción de este concepto no busca desviarlo de los factores y métricas establecidas de UX, sino más bien, enfocarlo para poder diseñar y evaluar la experiencia teniendo como objeto de estudio principal al usuario por lo que es, un conductor. Tratar al conductor como un usuario con atributos específicos de su campo de acción, que en este caso es hacer uso del vehículo, puede conducir a un mejor diseño de los componentes y estructura del automóvil, para así lograr una mejor interacción entre partes. Factores como (Carga de Trabajo Mental del Conductor, Confianza del Conductor, Estética del Vehículo, Usabilidad del Vehículo, Co-experiencia del Conductor, y Seguridad Percibida por el Conductor), pueden resultar en elementos fundamentales a la hora de diseñar una gran experiencia al conducir, por lo que deben ser considerados y contextualizados para darle al vehículo un uso más coherente, y permitir que tecnologías emergentes como los automóviles autónomos y los sistemas infotainment modernos sigan avanzando junto con la aceptación del conductor. Como trabajo futuro, encontramos una oportunidad para mejorar la experiencia del conductor al proponer un conjunto de patrones de diseño de interfaz de usuario para sistemas infotainment con base en puntos débiles detectados que podrían afectar los factores descritos en este enfoque de DX y que podrían ayudar a mejorar la relación vehículo-conductor.

\section{Declaración de conflicto de intereses}

Los autores declaran no tener conflicto de intereses con respecto a la investigación, autoría o publicación de este artículo.

\section{Referencias}

Alben, L. (1996). Quality of experience: defining the criteria for effective interaction design. Interactions, 3(3), 11-15. https://doi.org/10.1145/235008.235010

Battarbee, K. (2004). Co-experience: understanding user experiences in interaction [University of Art and Design Helsinki]. https://aaltodoc.aalto.fi/handle/123456789/11462

Blackwell, A. F., \& Fincher, S. (2010). PUX: patterns of user experience. Interactions, 17(2), $27-31$. https://doi.org/10.1145/1699775.1699782

Bloch, P. H. (1995). Seeking the Ideal Form: Product Design and Consumer Response. Journal of Marketing, 59(3), 16-29. https://doi.org/10.2307/1252116

Boehm-Davis, D. A., Green, P. A., Hada, H., Marcus, A., \& Wheatley, D. (2003). The next revolution: Vehicle userinterfaces and the global rider/driver experience. Conference on Human Factors in Computing Systems Proceedings, 708-709. https://doi.org/10.1145/765891.765941

Borchers, J. O., \& Thomas, J. C. (2001). Patterns: what's in it for HCI? CHI '01 extended abstracts on Human factors in computing systems - CHI '01, 225. https://doi.org/10.1145/634067.634201

Bosley, J. J. (2013). Creating a Short Usability Metric for User Experience (UMUX) Scale. Interacting with Computers, 25(4), 317-319. https://doi.org/10.1093/iwc/iwt007

Brooke, J. (1996). SUS: a "quick and dirty" usability scale. En P. W. Jordan, B. Thomas, B. A. Weerdmeester, \& I. L. McClelland (Eds.), Usability evaluation in industry (pp. 189-194). Taylor \& Francis Ltd.

Brookhuis, K. A., \& de Waard, D. (2010). Monitoring drivers' mental workload in driving simulators using physiological measures. Accident Analysis \& Prevention, 42(3), 898-903. https://doi.org/10.1016/j.aap.2009.06.001

Cobo, M. J., López-Herrera, A. G., Herrera-Viedma, E., \& Herrera, F. (2012). SciMAT: A new science mapping analysis software tool. Journal of the American Society for Information Science and Technology, 63(8), 1609- 
1630. https://doi.org/10.1002/asi.22688

da Silva, F. P. (2014). Mental Workload, Task Demand and Driving Performance: What Relation? Procedia - Social and Behavioral Sciences, 162, 310-319. https://doi.org/10.1016/j.sbspro.2014.12.212

Eggermeier, F. T., Gleen, W. F., Kramer, A. F., \& Damos, D. L. (1991). Workload assessment in multi-task environments. En D. L. Damos (Ed.), Multiple Task Performance (pp. 207-216).

Forlizzi, J., \& Battarbee, K. (2004). Understanding experience in interactive systems. Proceedings of the 2004 conference on Designing interactive systems processes, practices, methods, and techniques - DIS '04, 261. https://doi.org/10.1145/1013115.1013152

Gefen, Karahanna, \& Straub. (2003). Trust and TAM in Online Shopping: An Integrated Model. MIS Quarterly, 27(1), 51-90. https://doi.org/10.2307/30036519

Habib, K., Shalkamy, A., \& El-Basyouny, K. (2019). Investigating the Effects of Mental Workload on Highway Safety. Transportation Research Record: Journal of the Transportation Research Board, 2673(7), 619-629. https://doi.org/10.1177/0361198119846474

Hassenzahl, M., \& Tractinsky, N. (2006). User experience - a research agenda. Behaviour \& Information Technology, 25(2), 91-97. https://doi.org/10.1080/01449290500330331

Hellweger, S., \& Wang, X. (2015). What is User Experience Really: towards a UX Conceptual Framework. arXiv. http://arxiv.org/abs/1503.01850

International Organization for Standardization. (2018). ISO 9241-11:2018: Ergonomics of human-system interaction - Part 11: Usability: Definitions and concepts. https:/www.iso.org/obp/ui/\#iso:std:iso:9241:-11:ed-2:v1:en

Kaiser, T., Perterer, N., Meschtrcherjakov, A., \& Mirning, A. (2016). Car User Experience Patterns: A Pattern Collection in Progress. The Eighth International Conferenceon Pervasive Patterns and Applications.

Karapanos, E., Zimmerman, J., Forlizzi, J., \& Martens, J.-B. (2009). User experience over time. Proceedings of the 27th international conference on Human factors in computing systems - CHI 09, 729. https://doi.org/10.1145/1518701.1518814

Law, E. L.-C., van Schaik, P., \& Roto, V. (2014). Attitudes towards user experience (UX) measurement. International Journal of Human-Computer Studies, 72(6), 526-541. https://doi.org/10.1016/j.ijhcs.2013.09.006

Lee, J. D., \& See, K. A. (2004). Trust in Automation: Designing for Appropriate Reliance. Human Factors: The Journal of the Human Factors and Ergonomics Society, 46(1), 50-80. https://doi.org/10.1518/hfes.46.1.50 30392

Lewis, M., Sycara, K., \& Walker, P. (2018). The Role of Trust in Human-Robot Interaction. En H. A. Abbass, J. Scholz, \& D. J. Reid (Eds.), Foundations of Trusted Autonomy. Studies in Systems, Decision and Control (pp. 135-159). Springer, Cham. https://doi.org/10.1007/978-3-319-64816-3 8

Li, R., Chen, Y. V., Sha, C., \& Lu, Z. (2017). Effects of interface layout on the usability of In-Vehicle Information Systems and driving safety. Displays, 49, 124-132. https://doi.org/10.1016/j.displa.2017.07.008

Mayer, R. C., Davis, J. H., \& Schoorman, F. D. (1995). An Integrative Model Of Organizational Trust. Academy of Management Review, 20(3), 709-734. https://doi.org/10.5465/amr.1995.9508080335

Medina-Lopez, C., Marin-Garcia, J. A., \& Alfalla-Luque, R. (2010). Una propuesta metodológica para la realización de búsquedas sistemáticas de bibliografía (A methodological proposal for the systematic literature review). WPOM-Working Papers on Operations Management, 1(2), 13-30. https://doi.org/10.4995/wpom.v1i2.786

Michon, J. A. (1985). A Critical View of Driver Behavior Models: What Do We Know, What Should We Do? En L. Evans \& R. C. Schwing (Eds.), Human Behavior and Traffic Safety (pp. 485-524). Springer, Boston, MA. https://doi.org/10.1007/978-1-4613-2173-6_19

Norman, D., \& Nielsen, J. (2014). The Definition of User Experience (UX). https://www.nngroup.com/articles/definition-user-experience/

Orlovska, J., Wickman, C., \& Söderberg, R. (2018). Big data analysis as a new approach for usability attributes evaluation of user interfaces: an automotive industry context. En D. Marjanović, M. Štorga, N. Škec, S.;Bojčetić, \& N. Pavković (Eds.), DS 92: Proceedings of the DESIGN 2018 15th International Design Conference (pp. 1651-1662). https://doi.org/10.21278/idc.2018.0243

Pavlou, P. A., \& Gefen, D. (2004). Building Effective Online Marketplaces with Institution-Based Trust. Information Systems Research, 15(1), 37-59. https://doi.org/10.1287/isre.1040.0015

Raats, K., Fors, V., \& Pink, S. (2020). Trusting autonomous vehicles: An interdisciplinary approach. Transportation Research Interdisciplinary Perspectives, 7, 100201. https://doi.org/10.1016/j.trip.2020.100201

Ross, J. (2014). The Business Value of User Experience. http://www.infragistics.com/media/335732/the_business_value_of_user_experience-3.pdf

Shimizu, K. (2017). Technological development in automotive industry and transformation in corporate governance system. Journal of Governance and Regulation, 6(3), 46-54. https://doi.org/10.22495/jgr v6 i3 p5

Tscheligi, M. (2012). UX Design for vehicles [Conference presentation]. AutomotiveUI '12: International Conference on Automotive User Interfaces and Interactive Vehicular Applications. http://www.auto-ui.org/12/adjunctproceedings/t5-ux-design-vehicles-tscheligi.pdf

van Schaik, P., \& Ling, J. (2008). Modelling user experience with web sites: Usability, hedonic value, beauty and goodness. Interacting with Computers, 20(3), 419-432. https://doi.org/10.1016/j.intcom.2008.03.001

Wang, B., Hensher, D. A., \& Ton, T. (2002). Safety in the road environment: A driver behavioural response perspective. Transportation, 29(3), 253-270. https://doi.org/10.1023/A:1015661008598 